\title{
GLOBAL EXPONENTIAL ATTRACTORS FOR A CLASS OF ALMOST-PERIODIC PARABOLIC EQUATIONS IN $\mathbb{R}^{N}$
}

\author{
PIERRE-A. VUILLERMOT
}

(Communicated by Barbara L. Keyfitz)

\begin{abstract}
In this article, we construct global exponential attractors for a class of almost-periodic semilinear reaction-diffusion equations with Neumann boundary conditions on bounded regions of $\mathbb{R}^{N}$. The class of problems that we analyze here contains, in particular, Fisher's equations of population genetics.
\end{abstract}

Consider the class of real semilinear parabolic Neumann boundary value problems of the form

$$
\left\{\begin{array}{l}
u_{t}(x, t)=\Delta u(x, t)+s(t) g(u(x, t)), \quad(x, t) \in \Omega \times \mathbb{R}^{+}, \\
\operatorname{Ran}(u) \subseteq\left(u_{0}, u_{1}\right), \\
(\partial u / \partial \underset{\sim}{n})(x, t)=0, \quad(x, t) \in \partial \Omega \times \mathbb{R}^{+} .
\end{array}\right.
$$

In (1), $\Omega$ denotes an open connected bounded subset of $\mathbb{R}^{N}$ with smooth boundary $\partial \Omega$ and $N \in[2, \infty) \cap \mathbb{N}^{+}$, while $\Delta$ stands for Laplace's operator in the $x$-variable. Furthermore, $s: \mathbb{R}^{+} \rightarrow \mathbb{R}$ is the restriction to $\mathbb{R}^{+}$of a Bohr almost-periodic function on $\mathbb{R}$, which we shall also denote by $s$, while $g \in$ $\mathscr{C}^{(5)}(\mathbb{R}, \mathbb{R})$ possesses at least two zeroes $u_{0}$ and $u_{1}$ such that $g(u)>0$ for every $u \in\left(u_{0}, u_{1}\right)$, with the property that $g^{\prime}\left(u_{0}\right)>0$ and $g^{\prime}\left(u_{1}\right)<0$. Finally, $\operatorname{Ran}(u)$ denotes the range of $u$ and $n$ stands for the normalized outer normal vector to $\partial \Omega$.

Problems of the form (1) occur in various fields of sciences, such as the theory of nerve pulse propagation and population genetics [2-4]. It is then natural to ask whether there are conditions on the function $s$ such that every classical solution $(x, t) \rightarrow u(x, t)$ to problem (1) that exists globally in time stabilizes around a stable attractor as $t \rightarrow \infty$. It is the purpose of this article to show that this is indeed possible. We shall refer to the above properties of $g$ as being the following hypothesis:

We have $g \in \mathscr{C}^{(5)}(\mathbb{R}, \mathbb{R})$ and there exist $u_{0}, u_{1} \in \mathbb{R}$ such that (G) $\quad g\left(u_{0}\right)=g\left(u_{1}\right)=0, g^{\prime}\left(u_{0}\right)>0, g^{\prime}\left(u_{1}\right)<0$, and $g(u)>0$ for every $u \in\left(u_{0}, u_{1}\right)$.

Received by the editors November 8, 1990 and, in revised form, April 8, 1991.

1991 Mathematics Subject Classification. Primary 35K, 58B, 58F.

Key words and phrases. Almost-periodic parabolic equations, infinite-dimensional dynamical systems, global exponential attractors, explicit rates of decay.

The author is a Max-Planck Fellow in Mathematics. 
Now let $\mathbb{R}_{B}$ be the Bohr compactification of the real line [7]. We shall identify the real Bohr almost-periodic function $s$ of problem (1) with its uniformly continuous extension on $\mathbb{R}_{B}$, namely, $s \in \mathscr{C}\left(\mathbb{R}_{B}, \mathbb{R}\right)$. We shall also write

$$
\mu_{B}(s)=\lim _{l \rightarrow \infty} l^{-1} \int_{0}^{l} d \xi s(\xi)
$$

for the time average of $s$. Moreover, we shall assume that the following two hypotheses hold:

$\left(S_{1}\right)$ We have $\mu_{B}(s) \neq 0$ and $t \rightarrow \int_{0}^{t} d \xi \hat{s}(\xi)=0(1)$ as $|t| \rightarrow \infty$, where $\hat{s}=s-\mu_{B}(s)$.

$\left(S_{2}\right)$ The restriction of $s$ to $\mathbb{R}^{+}$is Hölder continuous.

Finally, we proceed to give the definition of a classical solution that we shall use throughout this article. Let $[N / 2]$ be the integer part of $N / 2$; in the re-

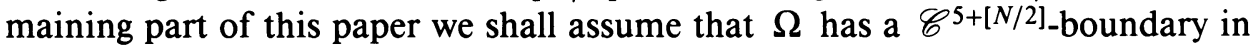
the sense of [1], in such a way that $\Omega$ lies on only one side of its boundary, and that is satisfies the interior ball condition for every $x \in \partial \Omega$ [5]. We denote by $\mathscr{C}^{2,1}\left(\Omega \times \mathbb{R}^{+}, \mathbb{R}\right)$ the set consisting of all functions $z \in \mathscr{C}\left(\Omega \times \mathbb{R}^{+}, \mathbb{R}\right)$ such that $(x, t) \rightarrow \partial_{t}^{\gamma} D^{\alpha} z(x, t) \in \mathscr{C}\left(\Omega \times \mathbb{R}^{+}, \mathbb{R}\right)$ for all $\alpha=\left(\alpha_{1}, \ldots, \alpha_{N}\right) \in \mathbb{N}^{N}, \gamma \in \mathbb{N}$, satisfying $\sum_{j=1}^{N} \alpha_{j}+2 \gamma \leq 2$. In a similar way we define $\mathscr{C}^{1,0}\left(\bar{\Omega} \times \mathbb{R}^{+}, \mathbb{R}\right)$. We then have

Definition 1. A function $u \in \mathscr{C}^{2,1}\left(\Omega \times \mathbb{R}^{+}, \mathbb{R}\right) \cap \mathscr{C}\left(\bar{\Omega} \times \mathbb{R}_{0}^{+}, \mathbb{R}\right) \cap \mathscr{C}^{1,0}\left(\bar{\Omega} \times \mathbb{R}^{+}, \mathbb{R}\right)$ is said to be a classical solution to problem (1) if the following conditions hold:

$\left(C_{1}\right)$ The partial derivative $(x, t) \rightarrow u_{t}(x, t)$ exists for every $t \in \mathbb{R}^{+}$uniformly in $x \in \bar{\Omega}$.

$\left(C_{2}\right) \quad x \rightarrow u(x, t) \in \mathscr{C}^{(2)}(\bar{\Omega}, \mathbb{R})$ for every $t \in \mathbb{R}^{+}$.

$\left(C_{3}\right)(x, t) \rightarrow u_{t}(x, t) \in \mathscr{C}\left(\bar{\Omega} \times \mathbb{R}^{+}, \mathbb{R}\right)$ and $t \rightarrow u_{t}(x, t) \in \mathscr{C}\left(\mathbb{R}^{+}, \mathbb{R}\right)$ uniformly in $x \in \bar{\Omega}$.

$\left(C_{4}\right) \quad u$ satisfies relations (1) identically.

The main result of this article then is

Theorem 1. Consider problem (1) where $g$ satisfies hypothesis (G). Assume, moreover, that $s$ satisfies hypotheses $\left(S_{1}\right)$ and $\left(S_{2}\right)$. Set $r_{u_{0}}=g^{\prime}\left(u_{0}\right) \mu_{B}(s)$, $r_{u_{1}}=g^{\prime}\left(u_{1}\right) \mu_{B}(s)$, and let $u$ be any classical solution to problem (1). Then there exist $\varepsilon_{0} \in(0, \infty), t_{\varepsilon_{0}} \in(0, \infty)$, and a positive constant $c_{0}$ such that the following statements hold:

(i) If $\mu_{B}(s)<0$, then the exponential decay estimates

$$
\sup _{x \in \bar{\Omega}}\left|u(x, t)-u_{0}\right| \leq c_{0} \varepsilon_{0} \exp \left[r_{u_{0}}\left(t-t_{\varepsilon_{0}}\right)\right],
$$

$$
\sup _{x \in \bar{\Omega}}|\nabla u(x, t)| \leq c_{0} \varepsilon_{0} \exp \left[r_{u_{0}}\left(t-t_{\varepsilon_{0}}\right)\right]
$$

hold for every $t \in\left[t_{\varepsilon_{0}}, \infty\right)$.

(ii) If $\mu_{B}(s)>0$, then a completely similar statement holds provided that we replace $u_{0}$ by $u_{1}$ everywhere in relations (3) and (4).

Remarks. (1) The above theorem asserts that if $\mu_{B}(s)<0$, then $u_{0}$ is a global exponential attractor for problem (1); every classical solution to problem (1) stabilizes around $u_{0}$ exponentially rapidly, with a rate of decay depending solely 
on $g^{\prime}\left(u_{0}\right)$ and $\mu_{B}(s)$, regardless of the spectral properties of Laplace's operator. We can thereby also conclude that the global stabilization phenomenon described above is primarily governed by the reaction process in (1). Of course, a similar remark holds if $\mu_{B}(s)>0$.

(2) Our result immediately implies that problem (1) has no time almostperiodic classical solution. For if $t \rightarrow u(x, t)$ were such a solution, relation (3) would immediately imply that $u(x, t)=u_{0}$ for every $(x, t) \in \bar{\Omega} \times \mathbb{R}^{+}$, a contradiction since $u_{0}$ is not a solution to problem (1).

(3) The simplest equation of the form (1) is the so-called Fisher's equation of population genetics, namely,

$$
\left\{\begin{array}{l}
u_{t}(x, t)=\Delta u(x, t)+s(t) u(x, t)(1-u(x, t)), \quad(x, t) \in \Omega \times \mathbb{R}^{+}, \\
\operatorname{Ran}(u) \subseteq(0,1), \\
(\partial u / \partial \underset{\sim}{n})(x, t)=0, \quad(x, t) \in \partial \Omega \times \mathbb{R}^{+} .
\end{array}\right.
$$

Under the conditions of the above theorem, every classical solution to problem (5) stabilizes exponentially rapidly toward $u_{0}=0$ or $u_{1}=1$, depending on whether $\mu_{B}(s)<0$ or $\mu_{B}(s)>0$. In the context of population genetics, (5) models, for instance, the evolution of the fraction $u$ of one of two alleles in the population of a migrating diploid species located in $\Omega$, when the so-called selection function $s$ takes almost-periodic seasonal variations into account. In this case, our result means that only one of the two alleles will eventually survive in the population.

(4) Our work concerning problem (1) was primarily inspired by the recent results of [6]. In fact, the authors of [6] obtained results concerning the case where $t \rightarrow s(x, t)$ is periodic and may also depend on $x \in \bar{\Omega}$; however, their method of proof seems to be strictly limited to the periodic case and does not provide the actual rates of decay.

The proof of the theorem rests upon the combination of a local geometric argument with a global one. We begin with the formulation of the local result. For $p \in(N, \infty)$, let $L^{p}(\mathbb{C})=L^{p}(\Omega, \mathbb{C})$ be the usual Lebesgue space with respect to Lebesgue measure on $\Omega$; define

$$
H_{\mathscr{N}}^{2, p}(\mathbb{R})=\left\{z \in H^{2, p}(\mathbb{R}):(\partial z / \partial \underset{\sim}{n})(x)=0, x \in \partial \Omega\right\}
$$

where $H^{2, p}(\mathbb{R})=H^{2, p}(\Omega, \mathbb{R})$ is the usual real Sobolev space of functions on $\Omega$. We may then assume that $H_{\mathscr{N}}^{2, p}(\mathbb{R})$ becomes a commutative Banach algebra with respect to the usual operations and the graph-norm [1]

$$
\|z\|_{\lambda_{0}, 2, p}=\left\|\left(\lambda_{0}-\Delta_{p, \mathscr{N}}\right) z\right\|_{p} .
$$

In relation $(7), \Delta_{p}, \mathscr{N}$ is the $L^{p}(\mathbb{C})$-realization of Laplace's operator whose domain is given by the complexification of $(6)$, while $\|\cdot\|_{p}$ denotes the usual $L^{p}$-norm and $\lambda_{0} \in \rho\left(\Delta_{p, N}\right)$, the resolvent set of $\Delta_{p, \mathscr{N}}$. Endowed with the norm (7), the above Banach algebra will henceforth be denoted by $H_{\lambda_{0}, \mathscr{N}}^{2, p}(\mathbb{R})$. Our local result is then the following proposition, which we also believe to be new. It amounts to constructing an infinite-dimensional stable manifold for problem (8).

Proposition 1. Let $s$ and $g$ satisfy all of the hypotheses of Theorem 1 and fix $p \in(N, \infty)$. Then there exist $\varepsilon_{1} \in(0, \infty), k_{1} \in[1, \infty)$ and, for each $\varepsilon \in$ 
$\left(0, \varepsilon_{1}\right]$, an open spherical neighborhood $\mathscr{N}_{\left(2 k_{1}\right)^{-1} \varepsilon}$ of radius $\left(2 k_{1}\right)^{-1} \varepsilon$ centered at the origin of $H_{\lambda_{0}, \mathscr{N}}^{2, p}(\mathbb{R})$ such that the following statements hold for every $t_{0} \in \mathbb{R}$ :

(i) If $\mu_{B}(s)<0$, and if we define $s_{t_{0}}(t)=s\left(t+t_{0}\right)$ for every $t \in \mathbb{R}$, then for each $\eta \in \mathscr{N}_{\left(2 k_{1}\right)^{-1} \varepsilon}^{+}=\left\{\eta \in \mathscr{N}_{\left(2 k_{1}\right)^{-1} \varepsilon}: \eta>0\right.$ on $\left.\bar{\Omega}\right\}$, there exists a classical solution $(x, t) \rightarrow \tilde{u}(x, t, \eta)$ to the problem

$$
\left\{\begin{array}{l}
u_{t}(x, t)=\Delta u(x, t)+s_{t_{0}}(t) g(u(x, t)), \quad(x, t) \in \Omega \times \mathbb{R}^{+}, \\
\operatorname{Ran}(u) \subseteq\left(u_{0}, u_{1}\right), \\
\left(\partial u / \partial \underset{\sim}{\sin }(x, t)=0, \quad(x, t) \in \partial \Omega \times \mathbb{R}^{+},\right.
\end{array}\right.
$$

which satisfies $\tilde{u}(x, 0, \eta)=\eta(x)+u_{0}$ for every $x \in \bar{\Omega}$. Moreover, the exponential decay estimates

$$
\begin{gathered}
\sup _{x \in \bar{\Omega}}\left|\tilde{u}(x, t, \eta)-u_{0}\right| \leq c_{1} \varepsilon \exp \left[r_{u_{0}} t\right], \\
\sup _{x \in \bar{\Omega}}|\nabla \tilde{u}(x, t, \eta)| \leq c_{1} \varepsilon \exp \left[r_{u_{0}} t\right]
\end{gathered}
$$

hold for every $t \in \mathbb{R}_{0}^{+}$and for some positive constant $c_{1}$.

(ii) If $\mu_{B}(s)>0$, then a completely similar result holds, provided that we replace $\mathscr{N}_{\left(2 k_{1}\right)^{-1} \varepsilon}^{+}$by $\mathscr{N}_{\left(2 k_{1}\right)^{-1} \varepsilon}^{-}=\left\{\eta \in \mathscr{N}_{\left(2 k_{1}\right)^{-1} \varepsilon}: \eta<0\right.$ on $\left.\bar{\Omega}\right\}$ and $u_{0}$ by $u_{1}$ everywhere in statement $(\mathrm{i})$.

For $t_{0}=0$, Proposition 1 provides particular classical solutions of small norm to problem (1) that satisfy exponential decay estimates similar to relations (3) and (4). In order to extend the validity of such estimates to all classical solutions of problem (1) and thereby obtain a proof of Theorem 1, we do need the arbitrariness of $t_{0}$ in Proposition 1 (compare with the proof of Theorem 1). For the sake of clarity, we postpone the proof of Proposition 1 until the end of the paper.

Proof of Theorem 1. Let $u$ be any classical solution to problem (1) and define $u(t)$ by $u(t)(x)=u(x, t)$ for every $(x, t) \in \bar{\Omega} \in \mathbb{R}^{+}$. From condition $\left(C_{2}\right)$ of definition 1 , it follows that $u(t) \in H_{\lambda_{0}, p}^{2, p}(\mathbb{R})$. If $\mu_{B}(s)<0$ then, upon using the remaining conditions of Definition 1 , hypothesis $\left(S_{2}\right)$, and the asymptotic theory for solutions to parabolic evolution equations [8], it follows that $u(t) \rightarrow$ $u_{0}$ strongly in $H_{\lambda_{0}, \mathcal{N}}^{2, p}(\mathbb{R})$ as $t \rightarrow \infty$. Consequently, all that we have to do is to wait long enough until $u(t)-u_{0}$ hits the stable manifold $\mathscr{N}_{\left(2 k_{1}\right)^{-1} \varepsilon}^{+}$at some time $t=t_{\varepsilon}$; for then we can identify $u(t)$ for $t \geq t_{\varepsilon}$ with a small norm-solution of Proposition 1 through the parabolic maximum principle. Thus fix $\varepsilon_{0} \in\left(0, \varepsilon_{1}\right)$; then there exists $t_{\varepsilon_{0}} \in(0, \infty)$ such that $\left\|u(t)-u_{0}\right\|_{\lambda_{0}, 2, p}<\left(2 k_{1}\right)^{-1} \varepsilon_{0}$ for every $t \in\left[t_{\varepsilon_{0}}, \infty\right)$. With this in mind, define $\eta_{0}=u\left(t_{\varepsilon_{0}}\right)-u_{0}$; since $u$ is a classical solution to problem (1), we may conclude that $\eta_{0} \in \mathscr{N}_{\left(2 k_{1}\right)^{-1} \varepsilon_{0}}^{+}$. It then follows from Proposition 1, with $t_{0}=t_{\varepsilon_{0}}$, that the boundary value problem

$$
\left\{\begin{array}{l}
u_{t}(x, t)=\Delta u(x, t)+s_{t_{\varepsilon_{0}}}(t) g(u(x, t)), \quad(x, t) \in \Omega \times \mathbb{R}^{+}, \\
\operatorname{Ran}(u) \subseteq\left(u_{0}, u_{1}\right), \\
(\partial u / \partial \underset{\sim}{n})(x, t)=0, \quad(x, t) \in \partial \Omega \times \mathbb{R}^{+},
\end{array}\right.
$$


possesses a classical solution $(x, t) \rightarrow \tilde{u}\left(x, t, \eta_{0}\right)$ that satisfies

$$
\tilde{u}\left(x, 0, \eta_{0}\right)=\eta_{0}(x)+u_{0}=u\left(x, t_{\varepsilon_{0}}\right)
$$

for every $x \in \bar{\Omega}$. Moreover, the exponential decay estimates

$$
\begin{gathered}
\sup _{x \in \bar{\Omega}}\left|\tilde{u}\left(x, t-t_{\varepsilon_{0}}, \eta_{0}\right)-u_{0}\right| \leq c_{1} \varepsilon_{0} \exp \left[r_{u_{0}}\left(t-t_{\varepsilon_{0}}\right)\right], \\
\sup _{x \in \bar{\Omega}}\left|\nabla \tilde{u}\left(x, t-t_{\varepsilon_{0}}, \eta_{0}\right)\right| \leq c_{1} \varepsilon_{0} \exp \left[r_{u_{0}}\left(t-t_{\varepsilon_{0}}\right)\right]
\end{gathered}
$$

hold for every $t \in\left[t_{\varepsilon_{0}}, \infty\right)$. We now define $(x, t) \rightarrow w(x, t)=\tilde{u}\left(x, t-t_{\varepsilon_{0}}, \eta_{0}\right)$ for every $(x, t) \in \bar{\Omega} \times\left[t_{\varepsilon_{0}}, \infty\right)$. It then follows from relations (11), (12) and the definition of $s_{t_{\varepsilon_{0}}}$ that $w$ satisfies the initial boundary value problem

$$
\left\{\begin{array}{l}
w_{t}(x, t)=\Delta w(x, t)+s(t) g(w(x, t)), \quad(x, t) \in \Omega \times\left(t_{\varepsilon_{0}}, \infty\right), \\
\operatorname{Ran}(w) \subseteq\left(u_{0}, u_{1}\right), \\
w\left(x, t_{\varepsilon_{0}}\right)=u\left(x, t_{\varepsilon_{0}}\right), \quad x \in \bar{\Omega}, \\
(\partial w / \partial \underset{\sim}{n})(x, t)=0, \quad(x, t) \in \partial \Omega \times\left(t_{\varepsilon_{0}}, \infty\right),
\end{array}\right.
$$

along with the exponential decay estimates (13) and (14). In order to complete the proof of Theorem 1(i), it thus remains to prove that $w(x, t)=u(x, t)$ for every $(x, t) \in \bar{\Omega} \times\left[t_{\varepsilon_{0}}, \infty\right)$. We first notice that $\operatorname{Ran}(u) \subseteq\left(u_{0}, u_{1}\right)$, $\operatorname{Ran}(w) \subseteq\left(u_{0}, u_{1}\right)$; moreover, $s$ is uniformly bounded on $\mathbb{R}$ and $g$ is smooth. The conclusion then follows from the third relation in (15) and the parabolic maximum principle. The proof of statement (ii), of course, is similar.

We conclude this article by providing a proof of Proposition 1 for the case $\mu_{B}(s)<0$. The case $\mu_{B}(s)>0$, of course, is similar. On $H_{\lambda_{0}, \mathcal{N}}^{2, p}(\mathbb{R})$, consider initial value problems of the form

$$
\left\{\begin{array}{l}
y^{\prime}(t)=\left(\Delta_{\mathcal{N}}+s_{t_{0}}(t) g^{\prime}\left(u_{0}\right)\right) y(t)+s_{t_{0}}(t) \hat{g}_{u_{0}}(y(t)), \quad t \in \mathbb{R}^{+}, \\
y(0)=y_{0},
\end{array}\right.
$$

where $s_{t_{0}}$ is as in the statement of Proposition 1. In expression (16), $\Delta_{\mathscr{N}}$ stands for the $H_{\lambda_{0}, \mathscr{N}}^{2, p}(\mathbb{C})$-realization of Laplace's operator on the domain

$$
\operatorname{Dom}\left(\Delta_{\mathscr{N}}\right)=\left\{z \in H_{\mathscr{N}}^{4, p}(\mathbb{C}): \Delta_{p, \mathscr{N}} z \in H_{\mathscr{N}}^{2, p}(\mathbb{C})\right\}
$$

where $H_{\lambda_{0}, \mathcal{N}}^{2, p}(\mathbb{C})$ and $H_{\mathcal{N}}^{4, p}(\mathbb{C})$ are the complexifications of $H_{\lambda_{0}, \mathcal{N}}^{2, p}(\mathbb{R})$ and $H_{\mathscr{N}}^{4, p}(\mathbb{R})$, respectively. Moreover, $\hat{g}_{u_{0}}: H_{\lambda_{0}, \mathcal{N}}^{2, p}(\mathbb{R}) \rightarrow H_{\lambda_{0}, \mathcal{N}}^{2, p}(\mathbb{R})$ is defined by

$$
\hat{g}_{u_{0}}(z)=g \circ\left(u_{0}+z\right)-g^{\prime}\left(u_{0}\right) z .
$$

Let $\left\{W_{\Delta_{\mathscr{N}}}(t)\right\}_{t \in \mathbb{R}_{0}^{+}}$be the restriction to $H_{\lambda_{0}, \mathcal{N}}^{2, p}(\mathbb{R})$ of the diffusion semigroup generated by $\Delta_{\mathscr{N}}$ on $H_{\lambda_{0}, \mathcal{N}}^{2, p}(\mathbb{C})$; define the family of evolution operators $\left\{U_{u_{0}, t_{0}}(t, r)\right\}_{t \geq r \geq 0}$ by

$$
U_{u_{0}, t_{0}}(t, r)=\exp \left[g^{\prime}\left(u_{0}\right) \int_{r}^{t} d \xi s_{t_{0}}(\xi)\right] W_{\Delta_{r}}(t-r) .
$$

It is easily verified that relation (19) with $r=0$ provides the evolution operators that solve the linear part of equation (16). 
Lemma 1. There exists a positive constant $c$, independent of $r, t$, and $t_{0}$, such that the exponential decay estimate

$$
\left\|\left|U_{u_{0}, t_{0}}(t, r)\right|\right\|_{\infty, \lambda_{0}, 2, p} \leq c \exp \left[r_{u_{0}}(t-r)\right]
$$

holds for the corresponding operator-norm.

Proof. Since $\mu_{B}\left(s_{t_{0}}\right)=\mu_{B}(s)$, we may write

$$
U_{u_{0}, t_{0}}(t, r)=\exp \left[r_{u_{0}}(t-r)\right] \exp \left[g^{\prime}\left(u_{0}\right) \int_{r}^{t} d \xi \hat{s}_{t_{0}}(\xi)\right] W_{\Delta_{\mathscr{N}}}(t-r)
$$

where $\hat{s}_{t_{0}}=s_{t_{0}}-\mu_{B}(s)$. But since $s$ satisfies hypothesis $\left(S_{1}\right)$, we have

$$
\int_{r}^{t} d \xi \hat{s}_{t_{0}}(\xi)=\int_{r}^{t} d \xi(\hat{s})_{t_{0}}(\xi)=0(1)
$$

uniformly in $r, t$, and $t_{0}$, where $(\hat{s})_{t_{0}}(t)=\hat{s}\left(t+t_{0}\right)$. The conclusion then follows from the fact that $\left\{W_{\Delta_{\mathscr{N}}}(t)\right\}_{t \in \mathbb{R}_{0}^{+}}$is a contraction semigroup on $H_{\lambda_{0}, \mathcal{N}}^{2, p}(\mathbb{R})$.

The preceding result suggests that we ought to be able to construct exponentially decaying classical solutions of small norm to problem (16). This is indeed the case.

Lemma 2. Let $g$ and $s$ satisfy the hypotheses of Theorem 1. Then there exist constants $\varepsilon_{1} \in(0, \infty), k_{1} \in[1, \infty)$ and, for each $\varepsilon \in\left(0, \varepsilon_{1}\right]$, an open spherical neighborhood $\mathscr{N}_{\left(2 k_{1}\right)^{-1} \varepsilon}$ of radius $\left(2 k_{1}\right)^{-1} \varepsilon$ centered at the origin of $H_{\lambda_{0}, \mathcal{N}}^{2, p}(\mathbb{R})$, such that the following statements hold for every $t_{0} \in \mathbb{R}$ :

(i) If $\mu_{B}(s)<0$, then for every $\eta \in \mathscr{N}_{\left(2 k_{1}\right)^{-1} \varepsilon}^{+}=\left\{\eta \in \mathscr{N}_{\left(2 k_{1}\right)^{-1} \varepsilon}: \eta>0\right.$ on $\bar{\Omega}\}$, there exists a unique function $t \rightarrow y(t, \eta) \in \mathscr{C}\left(\mathbb{R}_{0}^{+}, H_{\lambda_{0}, \mathfrak{N}}^{2, p}(\mathbb{R})\right) \cap$ $\mathscr{C}^{(1)}\left(\mathbb{R}^{+}, H_{\lambda_{0}, \mathcal{N}}^{2, p}(\mathbb{R})\right)$ that provides a classical solution to the Cauchy problem (16) with $y(0)=\eta$ and that satisfies the exponential decay estimate

$$
\|y(t, \eta)\|_{\lambda_{0}, 2, p} \leq \varepsilon \exp \left[r_{u_{0}} t\right]
$$

for every $t \in \mathbb{R}_{0}^{+}$.

(ii) If $\mu_{B}(s)>0$, then a similar statement holds if we replace everywhere $\mathscr{N}_{\left(2 k_{1}\right)^{-1} \varepsilon}^{+}$by $\mathscr{N}_{\left(2 k_{1}\right)^{-1} \varepsilon}^{-}=\left\{\eta \in \mathscr{N}_{\left(2 k_{1}\right)^{-1} \varepsilon}: \eta<0\right.$ on $\left.\bar{\Omega}\right\}$ and $u_{0}$ by $u_{1}$ in statement (i).

Proof. Let $Y_{r_{u_{0}}}$ be the real Banach space of all continuous mappings $y: \mathbb{R}_{0}^{+} \rightarrow$ $H_{\lambda_{0}, \mathcal{N}}^{2, p}(\mathbb{R})$ with respect to the usual pointwise operations and the norm $y \rightarrow$ $\|y\|_{r_{u_{0}}}=\sup _{t \in \mathbb{R}_{0}^{+}}\|y(t)\|_{\lambda_{0}, 2, p} \exp \left[-r_{u_{0}} t\right]<\infty$. By using the usual variation of constants formula, we can easily verify that the requirements $y \in Y_{r_{u_{0}}}$ and $y \in \mathscr{C}\left(\mathbb{R}_{0}^{+}, H_{\lambda_{0}, \mathcal{N}}^{2, p}(\mathbb{R})\right) \cap \mathscr{C}^{(1)}\left(\mathbb{R}^{+}, H_{\lambda_{0}, \mathcal{N}}^{2, p}(\mathbb{R})\right)$ imply that $y$ is a classical exponentially decaying solution to problem (16) if and only if the relation

$$
y(t)=U_{u_{0}, t_{0}}(t, 0) y_{0}+\int_{0}^{t} d \xi U_{u_{0}, t_{0}}(t, \xi) s_{t_{0}}(\xi) \hat{g}_{u_{0}}(y(\xi))
$$

holds for every $t \in \mathbb{R}_{0}^{+}$. Now for every such $t$, write

$$
F_{0}(y, \eta)(t)=U_{u_{0}, t_{0}}(t, 0) \eta
$$


for each $\eta \in H_{\lambda_{0}, \mathcal{N}}^{2, p}(\mathbb{R})$; furthermore, let

$$
F_{1}(y)(t)=\int_{0}^{t} d \xi U_{u_{0}, t_{0}}(t, \xi) s_{t_{0}}(\xi) \hat{g}_{u_{0}}(y(\xi)) .
$$

It follows immediately from Lemma 1 that $y \in Y_{r_{u_{0}}}$ implies $F_{0}(y, \eta) \in Y_{r_{u_{0}}}$. Now recall that $H_{\lambda_{0}, \mathcal{N}}^{2, p}(\mathbb{R})$ is a Banach algebra since $p \in(N, \infty)$ [1]. From this, we infer that the structure of our theory is similar to that developed in [9] for the analysis of some hyperbolic problems. In particular, we can easily verify that $\hat{g}_{u_{0}} \in \mathscr{C}^{(2)}\left(H_{\lambda_{0}, \mathcal{N}}^{2, p}(\mathbb{R}), H_{\lambda_{0}, \mathcal{N}}^{2, p}(\mathbb{R})\right)$ as a consequence of the differentiability hypothesis $g \in \mathscr{C}^{(5)}(\mathbb{R}, \mathbb{R})$ and that there exists a nondecreasing mapping $\Phi: \mathbb{R}_{0}^{+} \rightarrow \mathbb{R}_{0}^{+}$such that the estimate

$$
\left\|D^{2} \hat{g}_{u_{0}}(z)(h, k)\right\|_{\lambda_{0}, 2, p} \leq \Phi\left(\|z\|_{\lambda_{0}, 2, p}\right)\|h\|_{\lambda_{0}, 2, p}\|k\|_{\lambda_{0}, 2, p}
$$

holds for all $z, h, k \in H_{\lambda_{0}, \mathcal{N}}^{2, p}(\mathbb{R})$. Since we may write

$$
\hat{g}_{u_{0}}(y(\xi))=\int_{0}^{1} d s(1-s) D^{2} \hat{g}_{u_{0}}(s y(\xi)) y^{2}(\xi),
$$

it follows from relations (26), (20), and (28) that $y \in Y_{r_{u_{0}}}$ implies $F_{1}(y) \in Y_{r_{u_{0}}}$. Consequently, as in [9], we can conclude that the mapping $F(\cdot, \eta): Y_{r_{u_{0}}} \rightarrow$ $Y_{r_{u_{0}}}$ defined by $F(y, \eta)(t)=F_{0}(y, \eta)(t)+F_{1}(y)(t)$ is a contraction in the ball $S_{r_{u_{0}}}(\varepsilon)=\left\{y \in Y_{r_{u_{0}}}:\|y\|_{r_{u_{0}}} \leq \varepsilon\right\}$ for $\varepsilon \in\left(0, \varepsilon_{1}\right]$ and $\varepsilon_{1}$ sufficiently small, whenever $\|\eta\|_{\lambda_{0}, 2, p} \leq\left(2 k_{1}\right)^{-1} \varepsilon$ for some $k_{1} \in[1, \infty)$. We can then easily show that the corresponding unique fixed point $y(\cdot, \eta)$ possesses all of the required properties. This is true, in particular, if $\eta \in \mathscr{N}_{\left(2 k_{1}\right)^{-1} \varepsilon}^{+}$when $\mu_{B}(s)<0$, or if $\eta \in \mathcal{N}_{\left(2 k_{1}\right)^{-1} \varepsilon}$ when $\mu_{B}(s)>0$.

Proof of Proposition 1. If $\mu_{B}(s)<0$, define the function $(x, t) \rightarrow \tilde{u}(x, t, \eta)=$ $y(t, \eta)(x)+u_{0}$ where $y(\cdot, \eta)$ is the fixed point of Lemma 2 , and recall that there exists the continuous embedding $H_{\lambda_{0}, \mathcal{N}}^{2, p}(\mathbb{R}) \rightarrow \mathscr{C}^{1, \beta}(\mathbb{R})$, where $\mathscr{C}^{1, \beta}(\mathbb{R})$ denotes the Banach space of all real Hölder continuous functions on $\bar{\Omega}$ with Hölderian derivatives $D^{\alpha} z$ of exponent $\beta \in\left(0,1-p^{-1} N\right],|\alpha| \in[0,1]$ and the usual norm [1]. It follows from this, relations (16), (18), (23), and standard parabolic regularity theory that $\tilde{u}(\cdot, \cdot, \eta)$ satisfies relations $(9),(10)$, as well as the first and third relations of $(8)$ in the classical sense of Definition 1. As for the range condition $\operatorname{Ran}(u) \subseteq\left(u_{0}, u_{1}\right)$, it is sufficient to check that the relation $\tilde{u}(x, 0, \eta)=\eta(x)+u_{0} \in\left(u_{0}, u_{1}\right)$ holds for every $x \in \bar{\Omega}$, for then the general case follows from the parabolic maximum principle. In other words we must check that $u_{0}<\eta(x)+u_{0}<u_{1}$ for every $x \in \bar{\Omega}$. The left-hand side inequality holds because of the condition $\eta>0$; as for the inequality $\eta(x)=$ $|\eta(x)|<u_{1}-u_{0}$, it follows from the requirement $\|\eta\|_{\lambda_{0}, 2, p}<c^{-1}\left(u_{1}-u_{0}\right)$, where $c$ denotes the embedding constant in $H_{\lambda_{0}, \mathcal{N}}^{2, p}(\mathbb{R}) \rightarrow \mathscr{C}^{1, \beta}(\mathbb{R})$. This can always be achieved in choosing, if necessary, $\varepsilon_{1}$ smaller and $k_{1}$ bigger than the values selected in Lemma 2. If $\mu_{B}(s)>0$, then the argument is similar with $\tilde{u}(x, t, \eta)=y(t, \eta)(x)+u_{1}$ and $\eta<0$.

Remark. In the case where $\mu_{B}(s)=0$ and $\int_{0}^{t} d \xi s(\xi)=0(1)$ as $|t| \rightarrow \infty$, the stabilization phenomenon of the classical solutions to problem 1 is completely 
different from that described in Theorem 1. In particular, its analysis requires the elaboration of a suitable center manifold theory. The corresponding results will be published elsewhere.

\section{REFERENCES}

1. R. A. Adams, Sobolev spaces, Academic Press, London and New York, 1975.

2. D. G. Aronson and H. F. Weinberger, Nonlinear dynamics in population genetics, combustion and nerve pulse propagation, Partial Differential Equations and Related Topics (J. A. Goldstein, ed.), Lecture Notes in Math., vol. 446, Springer, Berlin, Heidelberg, and New York, 1975, pp. 5-49.

3. A. Fasano and M. Primicerio, Nonlinear diffusion problems, Lecture Notes in Math., vol. 1224, Springer, Berlin, Heidelberg, and New York, 1986.

4. P. C. Fife, Aspects of reacting and diffusing systems, Lecture Notes in Biomath., vol. 28, Springer, Berlin, Heidelberg, and New York, 1979.

5. D. Gilbarg and N. S. Trudinger, Elliptic partial differential equations of second order, Springer, Berlin, Heidelberg, and New York, 1983.

6. P. Hess and $\mathrm{H}$. Weinberger, Convergence to spatial temporal clines in the Fisher equation with time-periodic fitnesses, J. Math. Biol. 28 (1990), 83-98.

7. B. M. Levitan and V. V. Zhikov, Almost-periodic functions and differential equations, Cambridge Univ. Press, London and New York, 1982.

8. A. Pazy, Semigroups of linear operators and applications to partial differential equations, Appl. Math. Sci., vol. 44, Springer-Verlag, Berlin, 1983.

9. P. A. Vuillermot, Periodic soliton solutions to nonlinear Klein-Gordon equations on $\mathbb{R}^{2}$, Differential Integral Equations 3 (1990), 541-570.

Max-Planck-Institut für Mathematik, Gottfried-Claren-Strasse 26, D-5300 Bonn 3, GeRMANY 